\title{
Compact, Ti:sapphire based methane-stabilized optical molecular frequency comb and clock
}

\author{
Andrew Benedick, ${ }^{1, *}$ Dmitry Tyurikov², Mikhail Gubin ${ }^{2}$, Ruth Shewmon ${ }^{1}$, Issac Chuang ${ }^{1}$, \\ and Franz X. Kärtner ${ }^{1}$ \\ ${ }^{1}$ Department of Electrical Engineering and Computer Science, Massachusetts Institute of \\ Technology, 77 Massachusetts Avenue, Cambridge, MA 02139, USA \\ ${ }^{2}$ Quantum Radiophysics Division, P.N. Lebedev Physical Institute, 119991 Moscow GSP-1, \\ Leninsky Prospect, 53, Russia \\ *Corresponding author: andrew_b@mit.edu
}

A compact optical clock and frequency comb system is presented, based on a $1 \mathrm{GHz}$ octave spanning Ti:sapphire laser referenced to the $F_{2}^{(2)}\left(P(7), v_{3}\right)$ optical transition in methane at $3.39 \mu \mathrm{m}$. The reference methane transition is accessed by a stabilized HeNe laser in a compact transportable format. The output from the octave spanning Ti:sapphire laser is used to generate a mid-IR comb at the reference wavelength $3.39 \mu \mathrm{m}$. Direct comparison of the stabilized optical frequency comb with a ULE cavity stabilized diode laser at $674 \mathrm{~nm}$ shows an Allan Deviation of the frequency comb of $3 \times 10^{-14}$ in $20 \mathrm{~s}$.

OCIS codes: (320.7160) Ultrafast technology; (120.3940) Metrology; (140.7090)

Ultrafast lasers 
Over the last ten years, frequency combs have emerged as powerful tools for molecular and atomic spectroscopy [1-3], astronomical spectrograph calibration $[4,5]$, and as low phase noise radio frequency master oscillators[6]. Frequency combs in these and other applications are based on solid-state or fiber based femtosecond lasers phase locked to high precision optical frequency references. The ultimate precision in frequency comb stability is achieved using a single trapped ion as the frequency reference[3], though these are the most sophisticated but least compact types of systems. While it has been shown possible to distribute frequency stability to the $10^{-14}$ level over moderate distances using existing, unstabilized fiber optic networks [7], widespread distribution is still years away. Further, stabilization to more readily available standards such as GPS or cesium only gives high stability on timescales much longer than those presented here. Because of this, development of compact high stability frequency references such as those based on methane, and to a lesser extent iodine and acetylene, enables high stability frequency combs to be used both in research and industrial settings. The frequency comb system described here uses a commercially available methane stabilized HeNe laser [8] (FITRAN, Russia) along with a home built $1 \mathrm{GHz}$ repetition rate octave spanning Ti:sapphire laser[9] which in principle can be replaced by a commercially available laser of equivalent performance, resulting in a nearly turn-key system.

For achieving an optical clock and stabilized femtosecond laser frequency comb, we use an optical reference in the form of a methane stabilized HeNe laser similar to the laser described in [8] to stabilize the repetition rate [10]. Because of the octave spanning spectrum generated directly from the laser we are able to directly employ the f-2f self-referencing technique [1] to stabilize the carrier-envelope phase or frequency $f_{c e o}$, Fig 1. Simultaneous stabilization of $f_{\text {rep }}$ and $f_{\text {ceo }}$ allows the output of the Ti:sapphire laser to be used as an absolute frequency 
measurement device assuming that the frequency to be measured is known to within $f_{\text {rep }} / 2$ of its actual value.

The Ti:sapphire laser has a $1 \mathrm{GHz}$ repetition rate, and uses a $2.12 \mathrm{~mm}$ long Ti:sapphire crystal which has an absorption coefficient of $2.25 \mathrm{~cm}^{-1}$, Fig.1. All four mirrors are broadband, highly reflective double-chirped dispersion compensating mirror pairs (DCMs) [11], which overcompensate for the dispersion of the Ti:sapphire crystal and air path of the cavity. To correct the overcompensation of Group Delay Dispersion (GDD) provided by the DCMs, two pieces of Barium Fluoride are used at Brewster's angle, a $0.7 \mathrm{~mm}$ plate and a shallow angle wedge of $1.7 \mathrm{~mm}$ center thickness, which allow fine tuning of the overall cavity dispersion. The combination of the sapphire crystal, DCMs and the Barium Fluoride allow tuning of the average overall cold cavity GDD very close to $0 \mathrm{fs}^{2}$ between $600 \mathrm{~nm}$ and $1100 \mathrm{~nm}$. The Barium Fluoride wedge is coated on one side to provide nominally $4 \%$ output coupling from $650 \mathrm{~nm}$ to $1050 \mathrm{~nm}$, rising to more than $50 \%$ for shorter and longer wavelengths. Because the amplitude and phase of a coating in transmission obeys Kramer's Kronig relations and since the transmission profile is constant over most of the spectral range used, the GDD introduced by the output coupling is negligible. Mode locking can be achieved anywhere between $7.25 \mathrm{~W}$ and the $10.5 \mathrm{~W}$ limit of available pump power, and over this range of pump power the output spectrum is virtually constant with a maximum output power of $600 \mathrm{~mW}$ achieved with $10 \mathrm{~W}$ of pump power, Fig.2.

By sending a fraction of the Ti:sapphire output through a $20 \mathrm{~mm}$ long periodically poled Lithium-Niobate crystal with a $21 \mu \mathrm{m}$ poling period intrapulse Difference Frequency Generation (DFG) provides an offset free MID-IR frequency comb at 3.39 um for locking to the reference laser. To achieve good conversion efficiency, the beam is first reflected off of 5 DCM mirrors to ensure good temporal overlap of the spectral components which contribute most to the DFG 
process, $810 \mathrm{~nm}$ and $1064 \mathrm{~nm}$, which differs from earlier work by Foreman[10] due to the more symmetric output spectrum from the current laser. Because there is no offset frequency in the comb generated in the DFG process, stabilization of the DFG comb corresponds to direct stabilization of the frequency spacing between modes in the Ti:sapphire output spectrum, i.e. the repetition rate [12], separate from stabilizing the carrier-envelope offset-frequency.

From the DFG process we generate $\sim 200 \mathrm{nW}$ of power as measured in a $50 \mathrm{~nm}$ bandwidth, giving $\sim 150 \mathrm{pW}$ per mode assuming a uniform distribution. This DFG output is sent through a HeNe discharge tube which gives a further $20 \mathrm{~dB}$ of gain prior to heterodyning with the HeNe laser, ultimately giving a $30 \mathrm{~dB}$ SNR in a $100 \mathrm{kHz}$ bandwidth. Stabilization of the mode spacing of the Ti:sapphire laser is achieved by offset locking the DFG comb to the methane stabilized HeNe laser using a second HeNe laser as a transfer oscillator and sending the phase error signal to a piezo mounted mirror in the laser cavity. The mirror and piezo are mounted on a small copper cylinder which is massive enough to allow the majority of the displacement force generated by the piezo to result in displacement of the mirror, yet short enough that the lowest order compression mode of the cylinder is greater than $100 \mathrm{kHz}$ yielding a piezo/mount system which can support feedback bandwidths exceeding $60 \mathrm{kHz}$.

For the carrier-envelope offset frequency stabilization, the standard $f-2 f$ stabilization technique is used. Because of the spatial chirp of the output beam from the Ti:sapphire laser, and the large amount of power available at $570 \mathrm{~nm}$ and $1140 \mathrm{~nm}, 300 \mu \mathrm{W}$ and $2.5 \mathrm{~mW}$ both in $10 \mathrm{~nm}$ bandwidths respectively, a non-collinear Michelson type geometry is used to phase the $f$ and $2 f$ components at the detector. After proper phasing, both components are sent through a $1 \mathrm{~mm}$ thick BBO crystal for Type II phase matched second harmonic generation, the results of which are collected through a $10 \mathrm{~nm}$ bandpass filter centered at $570 \mathrm{~nm}$ and a beatnote of up to $50 \mathrm{~dB}$ in 
a $100 \mathrm{kHz}$ bandwidth is generated using an avalanche photodiode (MenloSystems APD210). This signal is phase locked to a high stability RF synthesizer by modulating the Ti:sapphire's pump power using an $\mathrm{AOM}$ which changes the intracavity pulse energy and subsequently the carrier-envelope frequency.

The entire frequency comb system described above consists of a minimal number of physical components suggesting the possibility of a truly compact system construction. The Ti:sapphire and all frequency stabilization optics fit comfortably on one $2 \mathrm{ft}$ by $3 \mathrm{ft}$ breadboard, while the reference laser is contained on another breadboard of the same size. The robust construction of the reference laser should allow stacking of the two optics boards to save table space if necessary with little to no degradation in performance. Electronics necessary for stabilization of the Ti:sapphire laser along with the pump laser controller and cooling system will fill only half of a standard size electronics rack.

The reference oscillator used in this work for measuring the Allan Deviation of the frequency comb was a $674 \mathrm{~nm}$ laser diode stabilized to a Fabry Perot cavity using the PoundDrever Hall locking method [13]. The cavity was constructed using a $10 \mathrm{~cm}$ long ultra low expansion glass spacer which was mounted to its support at the center of the spacer with the optical axis vertical to minimize the effect of ambient mechanical vibrations. Optically contacted mirrors provide a measured cavity finesse of 708,000+/-10,000.

The frequency comb and diode laser sources were located in the same building, but were separated by one floor and $\sim 30 \mathrm{~m}$ direct distance. Light generated by the stabilized diode was delivered to the Ti:sapphire comb by a single mode polarization maintaining fiber which was not stabilized due to the short length of the fiber $(60 \mathrm{~m})$ and the expected level of frequency stability to be measured [7]. A $10 \mathrm{~nm}$ bandwidth around $674 \mathrm{~nm}$ from the stabilized frequency comb 
gave $\sim 900 \mu \mathrm{W}$ was heterodyned against $\sim 800 \mu \mathrm{W}$ of light from the stabilized diode laser generating a $35 \mathrm{~dB}$ SNR radio frequency beat note at $\sim 18 \mathrm{MHz}$. This RF signal was counted using two zero dead time frequency counters (Pendulum CNT-90) to ensure that adequate signal to noise ratio was maintained during the measurement. The Allan Deviation plot in Fig.3 shows that the measured stability of the diode/frequency comb system as measured at $674 \mathrm{~nm}$ directly follows the stability of the methane stabilized reference laser, which was determined by a direct comparison of two identical HeNe lasers. For gate times longer than $20 \mathrm{~s}$ the Allan Deviation from the current measurement increases while the expected stability of the methane laser continues to decrease until about $100 \mathrm{~s}$ where long term pressure and temperature drifts take over. Drifts in the current measurement beginning at $20 \mathrm{~s}$ originate from the uncompensated temperature changes of the $674 \mathrm{~nm}$ diode laser's reference cavity.

For using the frequency comb in quantum information science or high speed sampling, pulse repetition rate phase noise is a primary concern. A preliminary characterization of high frequency phase noise of the optical clock was achieved by detecting the pulse repetition rate of the Ti:sapphire output using a GaAs photodiode, filtering the $6^{\text {th }}$ harmonic and amplifying the signal using a low noise amplifier before analyzing the signal using a Agilent E5052 Signal Source Analyzer. As is evident from Fig.4 the major noise contribution is from the white noise floor of the photodetection process and the noise floor of the Agilent signal source analyzer[14]. Despite this heavily instrument limited measurement, we are able to give a bound for the maximum timing jitter integrated from $10 \mathrm{MHz}$ to $40 \mathrm{~Hz}$ of only $10 \mathrm{fs}$.

We have presented a compact, transportable frequency comb system based on a $1 \mathrm{GHz}$ octave spanning Ti:sapphire laser and a commercially available methane stabilized HeNe laser, using a conceptually simple scheme for comb stabilization. The system simplicity is highlighted 
by the octave spanning output of the Ti:sapphire laser, the commercial availability and easy operability of the reference laser, and the separation of control of the Ti:sapphire's carrier envelope offset frequency and the pulse repetition rate. The high level of frequency stability provided by this compact and still transportable system is a distinct advantage enabling widespread deployment of visible frequency comb technology. 


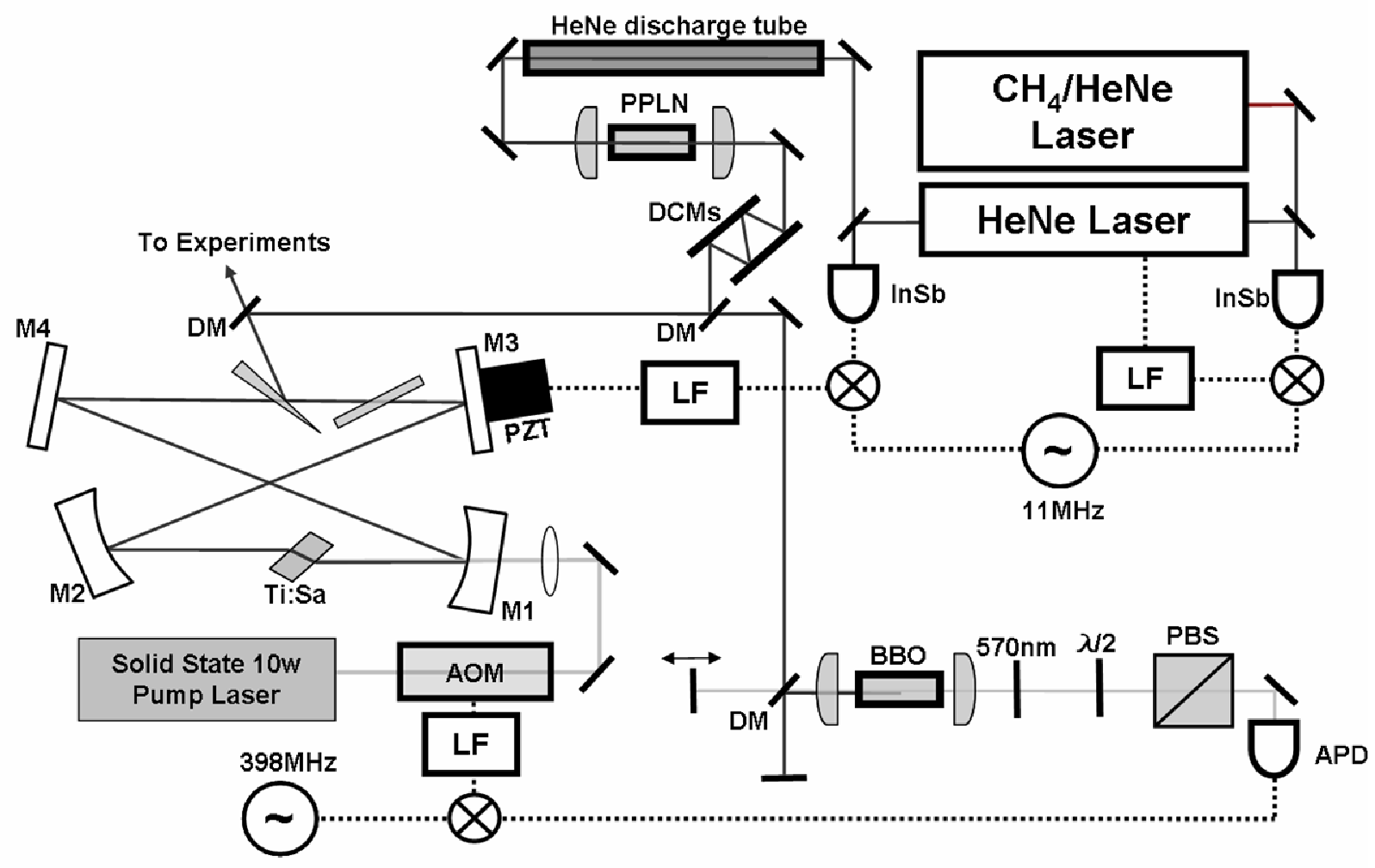

Fig. 1.

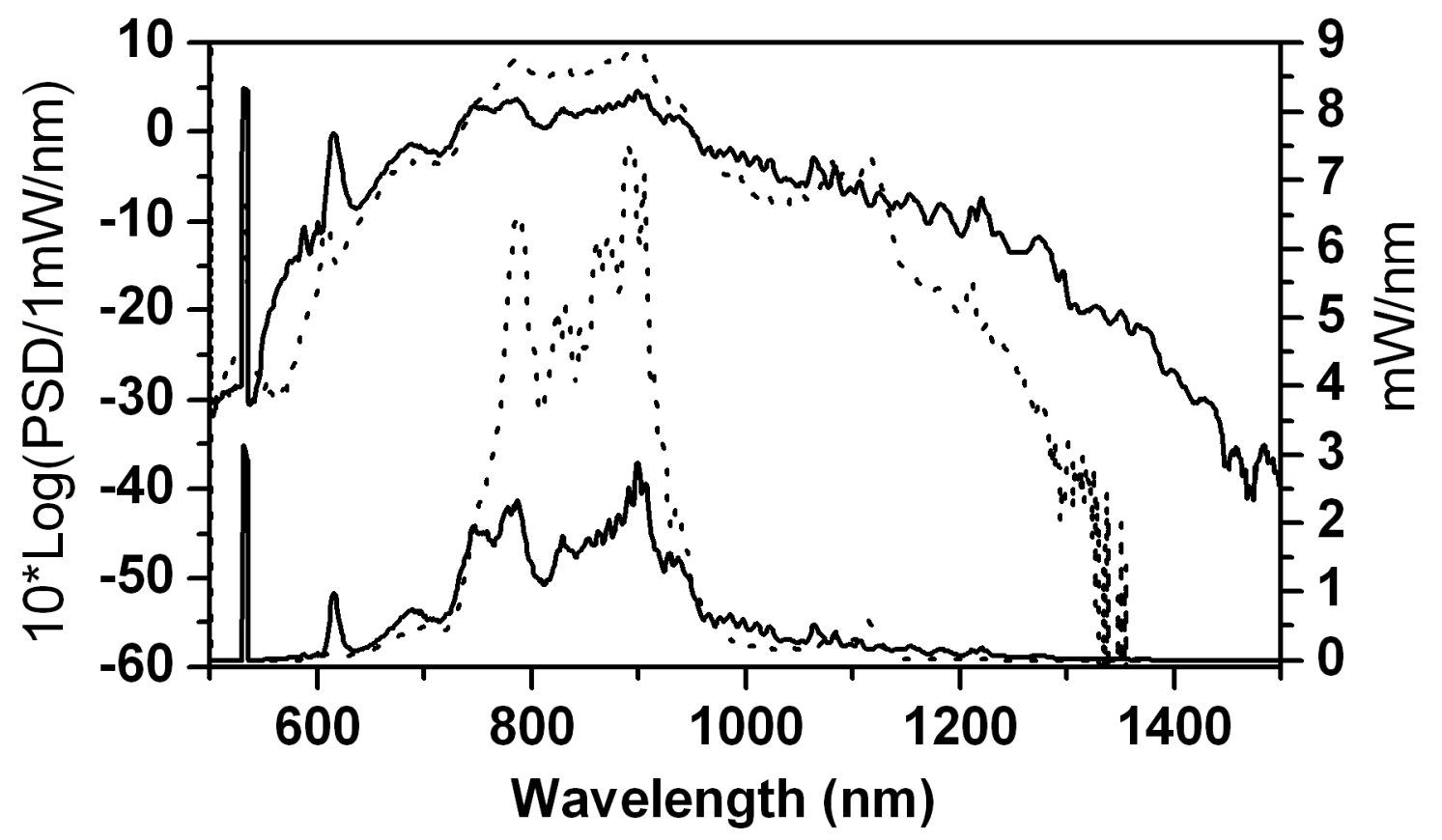

Fig. 2. 


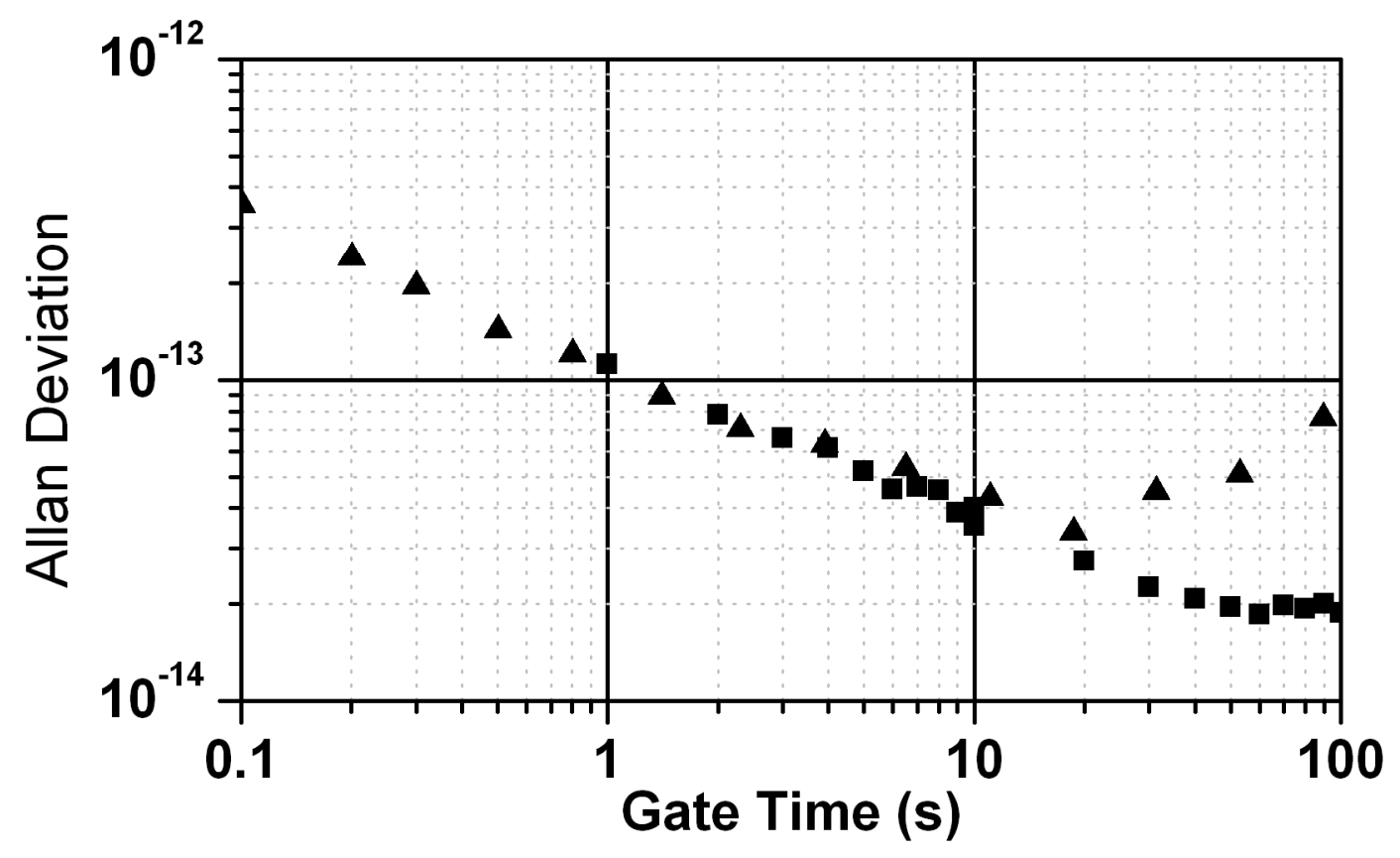

Fig. 3.

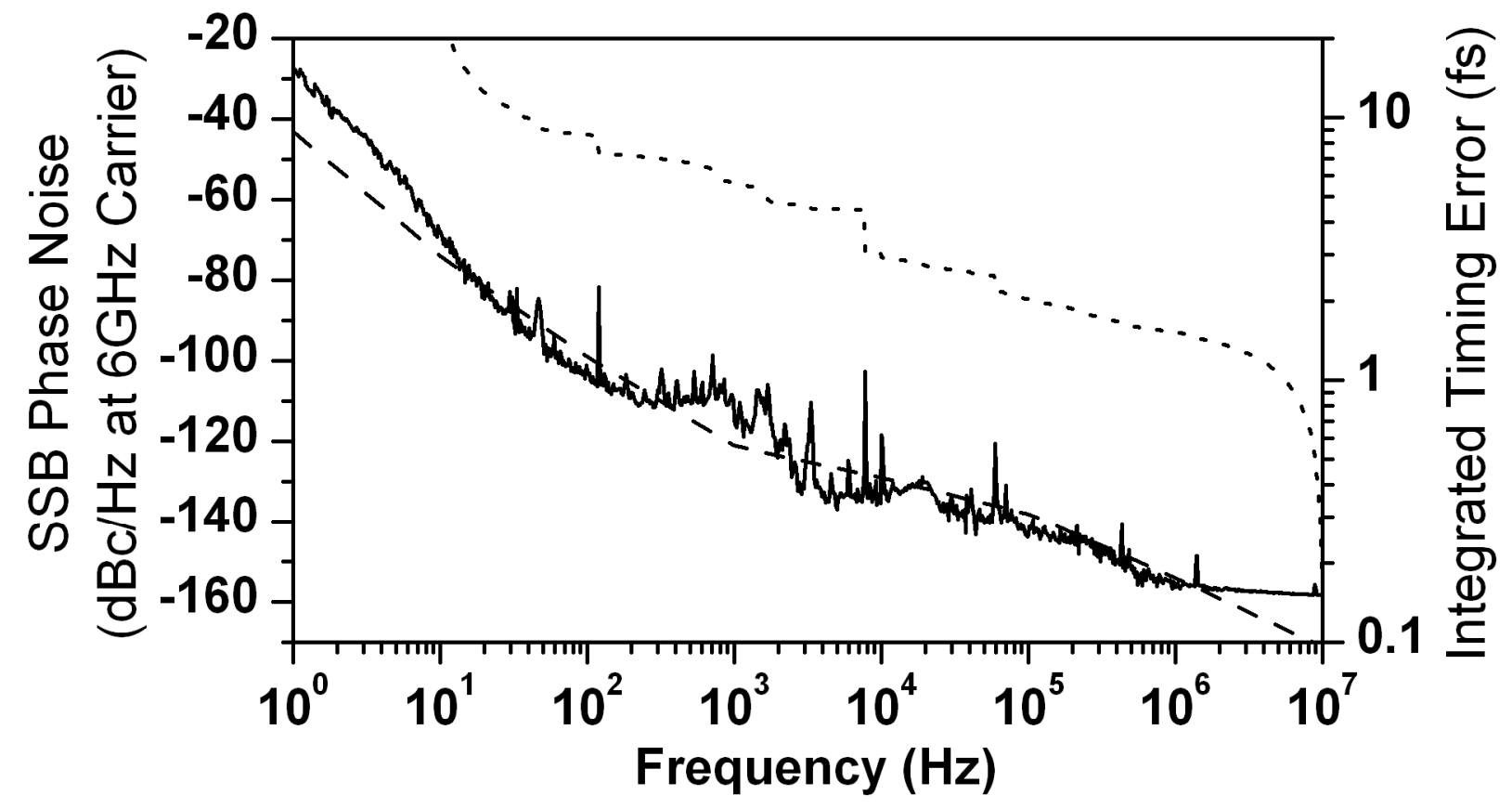

Fig.4. 
Fig. 1. Schematic of the $1 \mathrm{GHz}$ Ti:sapphire laser and $\mathrm{CH}_{4} / \mathrm{HeNe}$ referenced frequency comb system. Repetition rate control optics are in the upper right corner and $f_{c e o}$ stabilization optics are in the lower right. AOM, Acousto-optic modulator; LF, Loop filter; PZT, Piezo electric transducer; DM, Dichroic mirror.

Fig. 2. Output spectrum from the Ti:sapphire laser using output couplers with Barium Fluoride (600mW avg. output) and Fused Silica(>1W avg. output) substrates in solid and dotted lines, respectively. The difference in width of the two spectra and total output power is attributed to uncompensated TOD of the Fused Silica and lower surface quality of Barium Flouride, respectively.

Fig. 3. Measured Allan Deviation of the heterodyne beat note between the cavity stabilized diode laser and the Ti:sapphire frequency comb plotted in triangles, and squares plot the stability of the methane stabilized HeNe reference laser. The divergence of the two plots at $20 \mathrm{~s}$ gate time is due to long term drifts of the cavity stabilized diode laser.

Fig. 4. The solid line plots the single sided phase noise of the $6^{\text {th }}$ harmonic of the Ti:sapphire laser's pulse repetition rate, and the dotted line is the integrated timing jitter starting at $10 \mathrm{MHz}$. The dashed line is the published noise floor of the Agilent 5052 signal source analyzer used for this measurement. 
1. R. Holzwarth, T. Udem, T. W. Hansch, J. C. Knight, W. J. Wadsworth, and P. S. J. Russell, "Optical frequency synthesizer for precision spectroscopy," Physical Review Letters 85, 2264-2267 (2000).

2. M. J. Thorpe, K. D. Moll, R. J. Jones, B. Safdi, and J. Ye, "Broadband cavity ringdown spectroscopy for sensitive and rapid molecular detection," Science 311, 1595-1599 (2006).

3. T. Rosenband, D. B. Hume, P. O. Schmidt, C. W. Chou, A. Brusch, L. Lorini, W. H. Oskay, R. E. Drullinger, T. M. Fortier, J. E. Stalnaker, S. A. Diddams, W. C. Swann, N. R. Newbury, W. M. Itano, D. J. Wineland, and J. C. Bergquist, "Frequency Ratio of Al+ and $\mathrm{Hg}+$ Single-Ion Optical Clocks; Metrology at the 17th Decimal Place," Science 319, 1808-1812 (2008).

4. C.-H. Li, A. J. Benedick, P. Fendel, A. G. Glenday, F. X. Kartner, D. F. Phillips, D. Sasselov, A. Szentgyorgyi, and R. L. Walsworth, "A laser frequency comb that enables radial velocity measurements with a precision of $1 \mathrm{~cm} \mathrm{~s}(-1)$," Nature 452, 610-612 (2008).

5. D. A. Braje, M. S. Kirchner, S. Osterman, T. Fortier, and S. A. Diddams, "Astronomical spectrograph calibration with broad-spectrum frequency combs," European Physical Journal D 48, 57-66 (2008).

6. S. A. Diddams, A. Bartels, T. M. Ramond, C. W. Oates, S. Bize, E. A. Curtis, J. C. Bergquist, and L. Hollberg, "Design and control of femtosecond lasers for optical clocks and the synthesis of low-noise optical and microwave signals," Ieee Journal of Selected Topics in Quantum Electronics 9, 1072-1080 (2003). 
7. S. M. Foreman, K. W. Holman, D. D. Hudson, D. J. Jones, and J. Ye, "Remote transfer of ultrastable frequency references via fiber networks," Review of Scientific Instruments 78, - (2007).

8. M. A. Gubin, D. A. Tyurikov, A. S. Shelkovnikov, E. V. Kovalchuk, G. Kramer, and B. Lipphardt, "Transportable He-Ne/Ch4 Optical Frequency Standard and Absolute Measurements of Its Frequency," Ieee Journal of Quantum Electronics 31, 2177-2182 (1995).

9. T. M. Fortier, A. Bartels, and S. A. Diddams, "Octave-spanning Ti : sapphire laser with a repetition rate $>1 \mathrm{GHz}$ for optical frequency measurements and comparisons," Optics Letters 31, 1011-1013 (2006).

10. S. M. Foreman, A. Marian, J. Ye, E. A. Petrukhin, M. A. Gubin, O. D. Mucke, F. N. C. Wong, E. P. Ippen, and F. X. Kartner, "Demonstration of a HeNe/CH4-based optical molecular clock," Optics Letters 30, 570-572 (2005).

11. F. X. Kartner, U. Morgner, R. Ell, T. Schibli, J. G. Fujimoto, E. P. Ippen, V. Scheuer, G. Angelow, and T. Tschudi, "Ultrabroadband double-chirped mirror pairs for generation of octave spectra," Journal of the Optical Society of America B-Optical Physics 18, 882-885 (2001).

12. M. Zimmermann, C. Gohle, R. Holzwarth, T. Udem, and T. W. Hansch, "Optical clockwork with an offset-free difference-frequency comb: accuracy of sum- and difference-frequency generation," Optics Letters 29, 310-312 (2004).

13. R. Shewmon, "Coherent Manipulations of Trapped 88Sr+ using the 4D5/2 $\rightarrow 5 \mathrm{~S} 1 / 2$ Transition," (Massachusetts Institute of Technology, Cambridge, 2008). 
14. J. Kim, J. Chen, J. Cox, and F. X. Kärtner, "Attosecond-resolution timing jitter characterization of free-running mode-locked lasers," Opt. Lett. 32, 3519-3521 (2007). 